\title{
Development and analytical validation of a 25-gene next generation sequencing panel that includes the BRCA1 and BRCA2 genes to assess hereditary cancer risk
}

Thaddeus Judkins', Benoît Leclair ${ }^{1}$, Karla Bowles', Natalia Gutin¹, Jeff Trost ${ }^{1}$, James McCulloch', Satish Bhatnagar², Adam Murray ${ }^{1}$, Jonathan Craft ${ }^{1}$, Bryan Wardell ${ }^{2}$, Mark Bastian², Jeffrey Mitchell ${ }^{2}$, Jian Chen ${ }^{2}$, Thanh Tran², Deborah Williams ${ }^{2}$, Jennifer Potter ${ }^{2}$, Srikanth Jammulapati ${ }^{2}$, Michael Perry ${ }^{2}$, Brian Morris ${ }^{2}$, Benjamin Roa ${ }^{*}$ and Kirsten Timms ${ }^{2}$

\begin{abstract}
Background: Germline DNA mutations that increase the susceptibility of a patient to certain cancers have been identified in various genes, and patients can be screened for mutations in these genes to assess their level of risk for developing cancer. Traditional methods using Sanger sequencing focus on small groups of genes and therefore are unable to screen for numerous genes from several patients simultaneously. The goal of the present study was to validate a 25-gene panel to assess genetic risk for cancer in 8 different tissues using next generation sequencing (NGS) techniques.

Methods: Twenty-five genes associated with hereditary cancer syndromes were selected for development of a panel to screen for risk of these cancers using NGS. In an initial technical assessment, NGS results for BRCA1 and BRCA2 were compared with Sanger sequencing in 1864 anonymized DNA samples from patients who had undergone previous clinical testing. Next, the entire gene panel was validated using parallel NGS and Sanger sequencing in 100 anonymized DNA samples. Large rearrangement analysis was validated using NGS, microarray comparative genomic hybridization (CGH), and multiplex ligation-dependent probe amplification analyses (MLPA).

Results: NGS identified 15,877 sequence variants, while Sanger sequencing identified 15,878 in the BRCA1 and BRCA2 comparison study of the same regions. Based on these results, the NGS process was refined prior to the validation of the full gene panel. In the validation study, NGS and Sanger sequencing were $100 \%$ concordant for the 3,923 collective variants across all genes for an analytical sensitivity of the NGS assay of $>99.92 \%$ (lower limit of 95\% confidence interval). NGS, microarray CGH and MLPA correctly identified all expected positive and negative large rearrangement results for the 25-gene panel.
\end{abstract}

Conclusion: This study provides a thorough validation of the 25-gene NGS panel and indicates that this analysis tool can be used to collect clinically significant information related to risk of developing hereditary cancers.

Keywords: Next generation sequencing, Hereditary cancer, BRCA1, BRCA2

\footnotetext{
* Correspondence: broa@myriad.com

'Myriad Genetic Laboratories, Inc., Salt Lake City, Utah, USA

Full list of author information is available at the end of the article
} 


\section{Background}

Approximately $7 \%$ of breast and $11 \%$ to $15 \%$ of ovarian cancers are estimated to be due to germline DNA mutations [1-3]. Mutations in the BRCA1 and BRCA2 genes account for the majority of the mutations that increase risk for these cancers [1-3]. In addition to these two genes, mutations in several others, such as $M L H 1$ and $\mathrm{MSH} 2$, can also convey significant increases in risk for the development of malignancies in other hereditary cancer syndromes [4]. The detection of germline mutations in blood samples from patients can be extremely useful for identifying patients at high risk of developing a malignancy. This genetic information can be used to guide treatment discussion and genetic counseling for at-risk family members.

Sanger DNA sequencing has been the standard method of screening for genetic variants in clinical practice. In order to use this methodology, users are required to focus on small groups of genes that are selected based on a patient's unique risk factors and family history. Consequently, the utility of the Sanger method is limited when analyzing multiple genes from several patients simultaneously because tests for targeted genes often need to be conducted serially instead of simultaneously [5]. However, this upfront selectivity combined with incomplete follow-through in reflex testing may reduce the sensitivity of the testing overall.

Next generation sequencing (NGS) platforms provide an alternative to Sanger DNA sequencing that is more efficient for the analysis of large gene panels, allowing for more effective simultaneous screening of multiple genes [6]. This technique relies on multiplexed sample preparation followed by massive parallel sequencing and requires a significant informatics component for analysis [6]. Because multiple genes can be analyzed at once, delays in the acquisition of genetic data can be reduced [6]. Use of NGS could enable physicians to assess many genes associated with increased cancer risk at once, providing results in less time than required for several Sanger sequencing analyses to be conducted serially. However, a lack of standardization in sample preparation techniques, platforms, data analysis methods, variant classification, and clinical interpretation are significant challenges to the use of NGS platforms in clinical practice. To that end, guidelines and recommendations for NGS have been developed $[7,8]$.

An optimized and validated assay design is critical to maximizing the analytical sensitivity and specificity of NGS assays and ensuring high-quality interpretation to facilitate clinical decision-making. The development and analytical validation of a clinical NGS panel of 25 genes associated with hereditary cancers that can be screened simultaneously for maximal efficiency is presented here.

\section{Methods}

\section{Development of the 25-gene panel}

Twenty-five genes associated with hereditary cancer syndromes [9] were selected for development of a panel to screen for syndromes associated with 8 primary types of cancer (breast, ovarian, colon, endometrial, melanoma, pancreas, gastric, and prostate) using NGS. The genes included in the panel were: BRCA1, BRCA2 (hereditary breast and ovarian cancer syndrome); $M L H 1, M S H 2$, MSH6, PMS2, EPCAM - for large rearrangements of the last two exons only (Lynch syndrome); $A P C$ (familial adenomatous polyposis/attenuated familial adenomatous polyposis syndrome); MUTYH (MUTYH-associated colon cancer risk/MUTYH-associated polyposis syndrome); CDKN2A (melanoma-pancreatic cancer syndrome); PALB2, ATM (hereditary breast and pancreatic cancer risk); STK11 (Peutz-Jeghers syndrome), PTEN (PTEN hamartoma tumor syndrome); TP53 (Li-Fraumeni syndrome); $C D H 1$ (hereditary diffuse gastric cancer syndrome); BMPR1A, SMAD4 (juvenile polyposis syndrome); $B A R D 1$ (hereditary breast cancer risk); CHEK2 (hereditary breast, colorectal and prostate cancer risk); $C D K 4$ (melanoma cancer syndrome); NBN (hereditary breast and prostate cancer risk); $R A D 51 C, B R I P 1$ (hereditary breast and ovarian cancer risk); and $R A D 51 D$ (hereditary ovarian cancer risk) (Table 1). Most of these genes are associated with medical management guidelines from professional societies, such as NCCN, but some have currently only been associated with higher lifetime risks in published studies and do not have associated medical management guidelines. Variants were classified using methods consistent with American College of Medical Genetics and Genomics Guidelines [10].

DNA target preparation and enrichment were performed using the RainDance microdroplet polymerase chain reaction (PCR) system (RainDance Technologies, Billerica, Massachusetts). The RainDance microfluidic system combines microdroplets containing DNA and PCR reaction mix with microdroplets containing PCR primer sets. The use of dropletized primer/DNA combinations allowed for use of high numbers of primers in the library without primer-primer interactions. Custom primers were then arranged into multiplexes of 5 amplicons for efficient DNA usage and dropletized into the RainDance library.

A custom primer library to amplify gene regions of interest was designed using an iterative process. Sequencing regions of coding exons were identified (Table 1) and flanked by up to 20 bases of upstream and 10 bases of downstream intronic sequence to allow for variants that occurred in conserved, proximal splicing elements. Several extensions to cover potential mutations such as more distal, putative splicing mutations or other nonexonic mutations were included in the design [11,12]. A 
Table 1 Genes included in the 25 -gene NGS panel

\begin{tabular}{ll}
\hline Gene name & Transcript ID \\
\hline APC & NM_000038.5 \\
ATM & NM_000051.3 \\
BARD1 & NM_000465.3 \\
BMPR1A & NM_004329.2 \\
BRCA1 & NM_007294.3 \\
BRCA2 & NM_000059.3 \\
BRIP1 & NM_032043.2 \\
CDH1 & NM_004360.3 \\
CDK4 & NM_000075.3 \\
CHEK2 & NM_007194.3 \\
EPCAM & NM_002354.2 \\
MLH1 & NM_000249.3 \\
MSH2 & NM_000251.2 \\
MSH6 & NM_000179.2 \\
MUTYH (alpha5) & NM_001128425.1 \\
MUTYH (alpha3) & NM_001048171.1 \\
NBN & NM_002485.4 \\
P14ARF & NM_058195.3 \\
P16 & NM_000077.4 \\
PALB2 & NM_024675.3 \\
PMS2 & NM_000535.5 \\
PTEN & NM_000314.4 \\
RAD51C & NM_058216.2 \\
RAD51D & NM_002878.3 \\
SMAD4 & NM_005359.5 \\
STK11 & NM_000455.4 000546.5 \\
TP53 & NM \\
\hline
\end{tabular}

list of all regions was assembled and provided to RainDance Technologies for automated primer design with required design criteria, in this case Illumina 2X150 base-pair paired end sequencing, to determine optimal amplicon length, primer placement and primer tail sequences. Putative PCR priming sites were selected by RainDance Technologies using genome build hg19/ GRCh37 with custom software and compared automatically with public variant databases to avoid nonspecific priming and common single nucleotide polymorphisms. Primers which were designed against genes that are currently tested in this laboratory by Sanger sequencing were also manually compared against our own variant lists to avoid common sequence variations that might interfere with primer binding. The risk of sequence artifacts due to interfering variants at primer binding sites is reduced through amplicon tiling in all but the terminal primers of gene regions. With an average of $>5$ amplicons per exon there is enough redundancy for most sequence variants that might alter primer bindings in other amplicons to be detected. This library was then synthesized and tested for quality and reproducibility. Shortcomings were addressed through new primer design before the process was repeated.

This assay can be broadly divided into 3 parts: the sequencing portion via NGS; the large rearrangement (LR) detection via NGS dosage analysis, microarray $\mathrm{CGH}$, and multiplex ligation-dependent probe amplification analyses (MLPA); and informatics assisted data review and reporting, including variant classification (Figure 1). For the NGS assay, genomic DNA was extracted from blood samples by QIAsymphony using the DSP DNA Midi kit (Qiagen, Venlo, The Netherlands), and a fixed input of $5 \mu \mathrm{g}$ was fragmented to approximately $3 \mathrm{~kb}$ using sonication (SonicMan, Brooks Life Science Systems, Spokane, Washington). The reaction mix containing fragmented DNA and PCR mastermix was dropletized and merged with the droplets containing the primers

\begin{tabular}{|c|c|c|}
\hline NGS & Large rearrangements & Review and reporting \\
\hline $\begin{array}{l}\text { - } \text { DNA extraction } \\
\text { - } \text { RainDance emPCR for } \\
\text { sample enrichment } \\
\text { - } \text { LR-PCR for PMS2, CHEK2 } \\
\text { pseudogene regions } \\
\text { - Illumina HiSeq, MiSeq NGS } \\
\text { - Life Technologies } 3730 \times 1 \\
\text { Sanger } \\
\text { - Mutation confirmation } \\
\text { - Low coverage regions }\end{array}$ & $\begin{array}{l}\text { - DNA extraction } \\
\text { - Microarray CGH } \\
\text { - Detect and confirm LRs } \\
\text { - MLPA for PMS2, CHEK2 } \\
\text { - Gene vs pseudogene } \\
\text { - LR vs gene conversion } \\
\text { - NGS dosage }\end{array}$ & $\begin{array}{l}\text { - Informatics, LIMS workflow } \\
\text { - Variant classification } \\
\text { - Unusual case pathway } \\
\text { - Reporting }\end{array}$ \\
\hline
\end{tabular}

Figure 1 Components of the 25-gene panel. CGH, comparative genomic hybridization; emPCR, emulsion polymerase chain reaction; LIMS, laboratory information management system; LR, large rearrangement; MLPA, multiplex ligation-dependent probe amplification, NGS, next generation sequencing 
through a high throughput microfluidic emulsion PCR system using a RainDance Thunderstorm. The fragmented, genomic template for this step is limited so that amplification is digital for most reactions; roughly 40,000 reaction droplets were inoculated per patient to ensure that the final PCR products were consistent and normalized. The resulting emulsion underwent $55 \mathrm{cy}-$ cles of amplification on a Mastercycler Pro thermocycler (Eppendorf, Hamburg, Germany) after which the PCR products were bead-purified using AMPure XP (Beckman Coulter, Brea, California). A 6-nucleotide "barcode" tag, specific to each sample within a batch, and Illumina-specific sequencing adaptors were attached using secondary PCR. Purified products were then pooled and sequenced on the Illumina HiSeq 2500 NGS instrument (Illumina Inc., San Diego, California) to generate $2 \mathrm{X} 150$ base-pair paired end sequencing reads according to manufacturer instructions. All reagents were stored according to and used within the timeframe specified by manufacturer recommendations.

Portions of the PMS2 and CHEK2 genes have highly homologous pseudogenes. Therefore, target enrichment was modified to incorporate long-range PCR to ensure specificity to the genes of interest. Specific long-range amplicons (LRAs) were generated by primary PCR performed using LA Taq Hot Start (Takara Bio Inc., Otsu, Japan) on $50 \mathrm{ng}$ of genomic DNA. LRA products were diluted 1:10,000, and a second round of PCR was completed to amplify specific regions of interest and to attach barcode and sequencing adaptors to samples. Equal amounts of sample were combined and diluted to $2 \mathrm{nM}$ for sequencing using the Illumina MiSeq NGS instrument for 2X150 base-pair paired end sequencing reads. These data for individual patients generated on the HiSeq and MiSeq are recombined informatically during data analysis.

\section{NGS informatic data analysis}

Base calling was completed using Illumina Sequence Control Software with Real Time Analysis. Samples had quality scores assigned, and all sequence reads were trimmed to remove primers and sequencing bases below Q30 using an optimized Burrows-Wheeler approach. The open-source program JAligner (http://jaligner.source forge.net) was used to align the trimmed sequence reads to an internal set of reference sequences comprising the genes of this panel and their associated pseudogenes. The system automatically discarded all reads that matched pseudogene reference sequences as well as or better than the genes of interest.

All data were reviewed using in-house-designed review software. This software annotated allele frequency, assessed zygosity and performed quality metrics. To assess quality, Q scores and percent mapped reads were calculated. Using this method, the average depth of coverage was $>1000 \mathrm{X}$, with a minimum depth of coverage of 50X per base. By maintaining the average depth of coverage around 1000X to 2000X the number of bases with low (50X to 100X) coverage can be minimized. High depth of coverage becomes important when trying to assess dosage by amplicon quantitation as a method for detecting large rearrangements. Any region covered by NGS sequencing with a depth of coverage $<50 \mathrm{X}$ was repeated using Sanger sequencing. After alignment, sequence variants were called based on quantitative thresholds; bases called with a non-wild-type frequency of $<10 \%$ are attributed to noise, $30 \%$ to $70 \%$ are called heterozygous changes, and $90 \%$ to $100 \%$ are called homozygous changes (Figure 2). Bases in intermediate frequencies $(10 \%-30 \%$, or $70 \%-90 \%)$ are followed up by Sanger sequencing.

\section{Analysis of large rearrangements}

Large rearrangements were identified using quantitative dosage analysis of the data obtained from NGS. This approach relied on the digital nature of the droplet PCR process and required the comparison of trimmed, mapped amplicon read counts for all 96 samples in a batch. For these data, read counts of each amplicon were first normalized to the average read count of the sample. This ratio was then normalized to adjust for variability across the batch of 96 samples. Next, all of the amplicons that overlapped an exon or region of interest were combined together into a summary value. Finally, a ratio for each region of interest was generated for each sample relative to all others within the batch and plotted as a scatterplot. This analysis was done using in-house-developed review software by trained data reviewers (Figure 3).

Identification of large rearrangements, specifically deletions and duplications, was also performed using additional methods to complement NGS. Twenty-three genes in the panel were assessed using a custom microarray comparative genomic hybridization (CGH) chip (Agilent Technologies, Santa Clara, California). A total of 349 regions, mainly coding exons along with $100 \mathrm{nu}-$ cleotides of flanking sequences, were covered by $>9,400$ custom oligonucleotide probes for an average coverage of 27 probes per region. Patient genomic DNA was fragmented and labeled with Cy5, and reference DNA was labeled with $\mathrm{Cy} 3$ using a custom version of the Agilent SureTag Labeling Kit. Labeled DNA was then combined and hybridized to a microarray containing oligonucleotide probes to gene regions. After hybridization, slides were washed to remove excess hybridization reagents and non-hybridized DNA. Scanning of washed slides was completed using an Agilent Microarray Slide Scanner, and data were extracted using Agilent Feature Extraction 


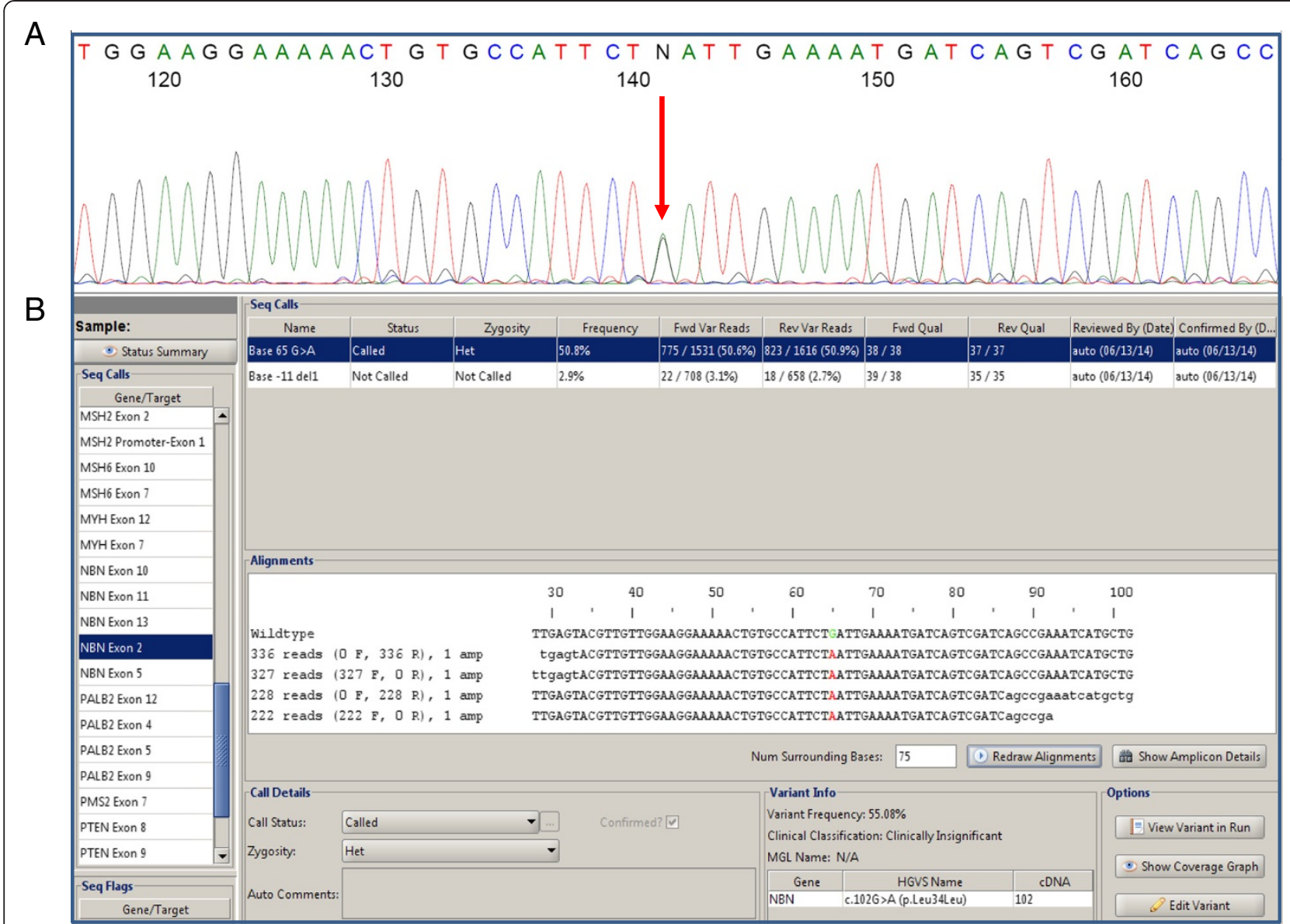

Figure 2 The same variant in NBN as detected by Sanger sequencing (A) and NGS (B). Note that the frequency of alleles at variable positions, read depth in independent forward and reverse reads and quality scores can be reviewed here. The heterozygous base change indicated by the arrow in panel $\mathbf{A}$ is the same base change selected in the NGS results in panel $\mathbf{B}$. NBN, NBN-associated cancer risk ; NGS, next generation sequencing.

Software. The analysis was performed by in-house-developed software using sample dosage normalization, locally weighted scatterplot smoothing normalization, historic probe normalization, and custom GC normalization (Figure 3).

MLPA analysis for large rearrangements in PMS2 and CHEK2 was conducted using probe mixes P008 and P190, respectively, from MRC-Holland (Amsterdam, The Netherlands). Pairs of adjacent oligonucleotide probes were hybridized to regions of interest, usually one probe pair in or near each exon. To improve specificity, probes were designed over gene-specific variants, to distinguish between homologous pseudogenes and the actual gene regions of PMS2 and CHEK2. Adjacent probes were ligated and then amplified by PCR using fluorescently labeled composite primers that included genomic target and stuffer sequences to differentiate products by size. Gene dosage results were analyzed using GeneMarker software (Softgenetics, State College, Pennsylvania).

\section{Validation procedures}

The 25-gene panel was validated using samples submitted for single-syndrome clinical testing to a Clinical Laboratory Improvement Amendments (CLIA) and College of American Pathology (CAP) approved laboratory. All patients who received clinical testing gave informed consent for testing and were over the age of 18 . Upon completion of clinical testing, all samples were anonymized for research by Myriad's Quality Assurance department. Any samples originating from states with legislation mandating destruction of biospecimens after completion of genetic testing were excluded. As a retrospective study performed on anonymized samples, this analytical validation was not subject to any additional review (HHS regulation 45 CFR 46). The sequencing component of the NGS panel was validated by comparing NGS with Sanger sequencing results in 100 samples. To facilitate this, Sanger primer sets were designed and tested for all exons sequenced by the NGS panel. 

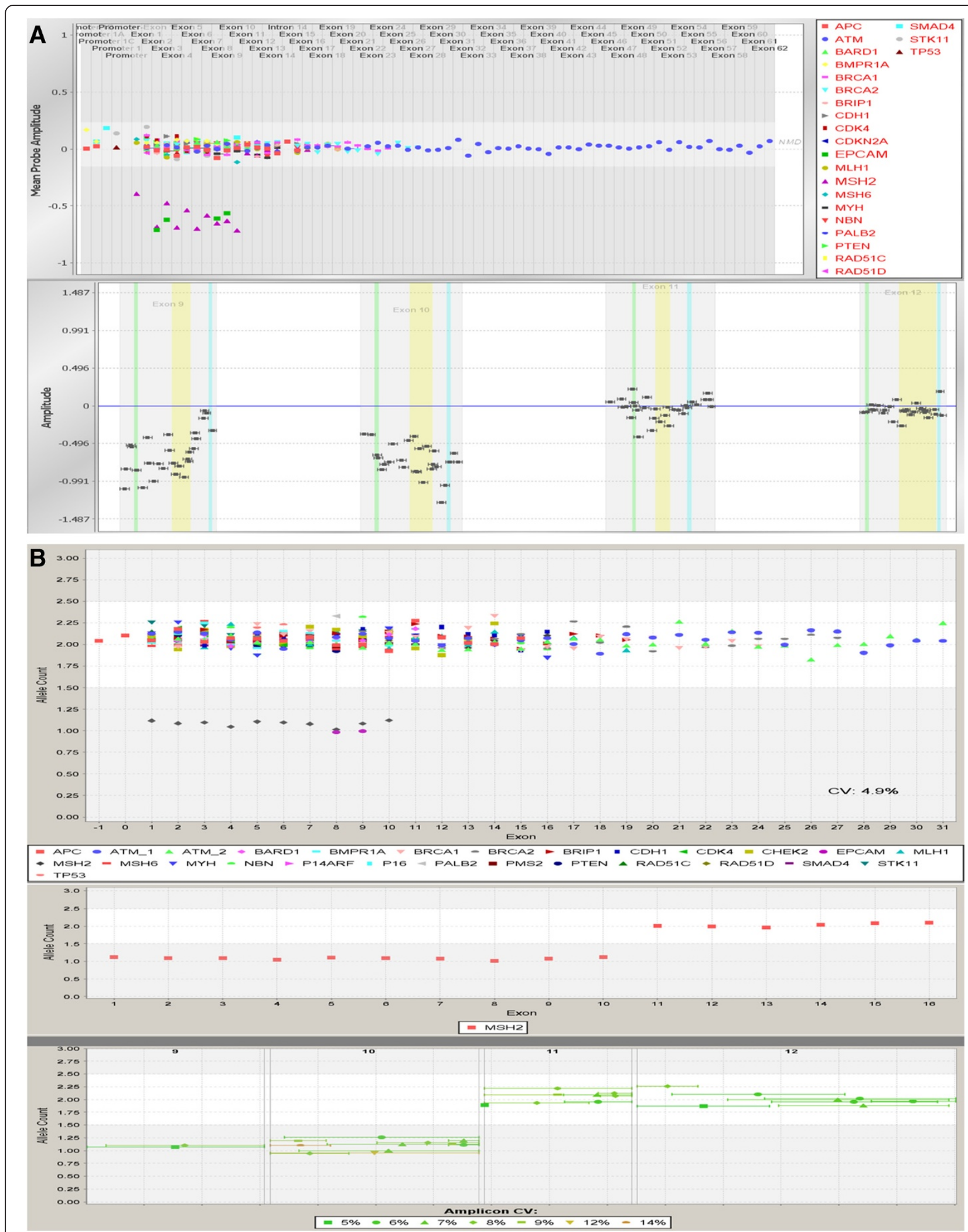

Figure 3 (See legend on next page.) 
(See figure on previous page.)

Figure 3 A contiguous deletion involving EPCAM and MSH2 as seen in both microarray CGH (A) and NGS LR (B) views. In the case of the microarray CGH plot the data are on a $\log _{2}$ scale with wild-type dosage at 0 on the $Y$-axis. In the case of the NGS dosage plot, the data are on a linear scale with wild-type dosage at 2 on the Y-axis. In both cases, a summary overview is available (top) as well as a zoomed in (bottom) view showing specific probe or amplicon placement. In the summary view, all genes are shown simultaneously with a symbol representing each gene, and exons proceeding $5^{\prime}$ to $3^{\prime}$ across the X-axis. Note that EPCAM exons 2-3 are also included in the microarray CGH assay for normalization purposes but are not tested on the NGS LR assay. NGS LR, next generation sequencing large rearrangements.

Large rearrangement analysis components of the 25gene panel were validated on additional positive samples, along with the 100 samples used for the sequencing portion of the validation which did not contain large rearrangement mutations. Deletion/duplication analyses on 23 genes were also validated by microarray CGH in 212 anonymized DNA samples and by NGS dosage analysis on a subset of 110 of these anonymized samples with sufficient volume. Genomic positive controls were supplemented with synthetic controls for microarray CGH. MLPA was validated for PMS2 and CHEK2 in 110 anonymized DNA samples. This set of 110 anonymized samples is the same set that was used in the microarray $\mathrm{CGH}$ and NGS dosage analysis validations.

\section{Statistical methods}

Sensitivity is the proportion of the number of true positives over the sum of the number of true positives and false negatives. It is the ability to correctly identify a sequence change from the wild type if that change exists. Specificity is the proportion of the number of true negatives over the sum of the number of true negatives and false positives. It is the ability to correctly exclude a sequence change from the wild type if that change does not exist.

Lower 95\% confidence bounds for sensitivity and specificity estimates were calculated using Minitab version 15, 1 proportion test, with the Exact method. Note that Minitab only calculates a 1-sided confidence interval (CI) when the numerator of the proportion equals the denominator because the upper bound is 100\%. By reporting the lower limit of the $95 \% \mathrm{CI}$, one can claim with $95 \%$ confidence that the actual sensitivity or specificity meets or exceeds the stated sensitivity or specificity.

\section{Results}

Initial sensitivity and specificity assessment of BRCA1 and $B R C A 2$ sequencing

For the initial sequencing assessment, NGS identified 15,877 variants, while prior Sanger sequencing identified 15,878 variants among 1864 anonymized samples from patients who had previously undergone BRCA1 and $B R C A 2$ testing. Patients were selected based on personal history that included cancer, though not limited to breast or ovarian malignancies. Of these variants, 3.02\% were deleterious or suspected deleterious mutations while only $0.67 \%$ were variants of uncertain significance. The results showed an estimated analytical sensitivity for NGS $>99.96 \%$ (lower limit of the 95\% CI) and an estimated analytical specificity $>99.99 \%$ (lower limit 95\% CI) for $B R C A 1$ and BRCA2 sequencing. One polymorphism, $B R C A 2$ c.7806-14C > T, was missed by NGS, determined to be caused by non-amplification of a PCR allele. The missed polymorphism was in a DNA sample with a rare co-occurring 3-base intronic deletion in a PCR primer binding site (BRCA2 c.7806-64_7806-62del), which has a frequency of approximately $0.05 \%$. No other differences in mutations detected were observed, including the 301 indels and 15,577 single-base substitutions identified here, or sensitivity for different types of mutations between Sanger sequencing and NGS.

Sensitivity of subsequent NGS data analysis was enhanced by using individual amplicon dosage to assess PCR allele dropout due to rare sequence variants under primer binding sites. First, the dosage of each amplicon relative to the rest of the amplicons in the sample is examined. Next the dosage is compared with the standard deviation of the amplicon across the whole batch. Based on these data, any amplicon that appears amplified from a single allele can be flagged. Flagged regions can be assessed through follow-up Sanger sequencing. This improvement was made possible by the enhanced quantitative nature of NGS that was not an option with Sanger sequencing. PCR-based sample preparation for both NGS and Sanger sequencing shares the same risk of variants interfering with primer binding but NGS allows for better detectability. Based on these initial positive results, the 25-gene NGS panel underwent further validation.

\section{Analytical validation of the 25 -gene NGS panel for clinical testing}

At this point changes to the assay were completed and validation was performed for the sequencing and large rearrangement components for the entire 25-gene panel test. NGS and Sanger sequencing were performed in parallel on 100 anonymized DNA samples. Sequencing results were $100 \%$ concordant for the 3923 collective variants identified in 100 DNA samples (Table 2). This included 3884 single base substitutions and 39 indels. The 39 indels included 4 insertions, 34 deletions, and one insertion coupled with a deletion. Of these, 20 
Table 2 Sanger and next generation sequencing results

\begin{tabular}{lll}
\hline & Sanger sequencing & NGS \\
\hline Samples & 100 & 100 \\
Amplicons (per sample) & 370 & 1969 \\
Bases analyzed (per sample) & 88,631 & 88,631 \\
Total positive bases & 3923 & 3923 \\
- Single base substitutions & 3884 & 3884 \\
-Small indels & 39 & 39 \\
Total negative bases & $8,859,177$ & $8,859,177$ \\
\hline
\end{tabular}

occurred in coding exons and 19 occurred in intronic regions flanking the exons that are involved in splicing. Analytical sensitivity of the NGS assay was estimated to be $>99.92 \%$ (lower limit of $95 \%$ CI). Analytical specificity of the NGS assay was estimated to be $>99.99 \%$ (lower limit of 95\% CI). Reproducibility studies were also performed, wherein 4 DNA samples were run in triplicate per batch, across 3 different batches. The data showed $100 \%$ concordant calls, which demonstrated intra-batch and inter-batch reproducibility.

The large rearrangement component of the assay was validated using an additional set of 212 anonymized DNA samples with known large rearrangement genotypes. Deletion/duplication analysis on 23 of the 25 genes in the panel was performed by microarray $\mathrm{CGH}$, which correctly identified all 51 genomic positive controls, including replicates and reproducibility controls, across different genes among the 212 anonymized DNA samples (Table 3). A partial set of 110 of these samples with sufficient volume, including 49 of the positive samples, was also processed using NGS for large rearrangement dosage analysis. Of the 49 large rearrangement positive samples processed, 48 produced results which were all concordant with the expected sample large rearrangements. The sample containing the final LR, a MSH2 deletion of exons 1-6, did not successfully complete laboratory processing and did not undergo data analysis. For some genes where rare genomic positive controls were not available, we supplemented the validation studies with synthetic positive controls created with restriction enzyme digests of genomic DNA for microarray CGH analysis. The results were consistent with simulated deletions in the affected regions. In addition, deletion/duplication analysis for the pseudogene-containing PMS2 and CHEK2 genes was validated using MLPA on 110 anonymized DNA samples with known genotypes. MLPA correctly identified all 5 genomic positive controls in PMS2 and CHEK2 among these 110 anonymized DNA samples.

\section{Discussion}

Recent advances in NGS and sample enrichment technologies allow for simultaneous assessment of multiple genes. Hereditary cancer panels have been constructed incorporating genes underlying well characterized cancer syndromes, such as BRCA1 and BRCA2, along with more recently discovered genes associated with increased cancer risk [13]. The use of gene panels in hereditary cancer risk assessment is increasing and studies to assess the prevalence of mutations among patients commonly referred for genetic testing have recently been published $[9,14,15]$. Additional studies are underway to more fully define the benefits and limitations of panel testing in the clinical setting. Analytical validation is a critical step in the laboratory development process to facilitate the availability of highly robust and reproducible clinical tests. The current study demonstrates the analytical validation of a 25-gene hereditary cancer panel by comparing results of Sanger sequencing and other methods to results of the NGS assay.

Initial analysis of $B R C A 1$ and $B R C A 2$ gene variants facilitated the optimization of this NGS assay that allowed for validation of a comprehensive 25-gene panel. Process improvements in NGS data analysis based on these initial results were able to enhance the sensitivity of the assay for detection of low coverage regions that were suggestive of potential deletions or PCR allele dropouts. This initial analysis also supported the standardized NGS methodology so that it could then be applied to the collection of mutation data that could be clinically significant using the 25 -gene panel. This is important because NGS is being used clinically, but there continue to be challenges in standardizing the different components that include the analytical wet bench process (sample preparation, target enrichment, NGS sequencing) and the bioinformatics pipeline for NGS [7]. Therefore, it is important for each lab to demonstrate a robust validation of their clinical NGS assays.

The validation of the gene panel presented here offers the opportunity to test a system that incorporates laboratory information management system tracking for all steps through the lab and custom software to support timely and accurate analysis of clinical data. Furthermore, it allowed testing and validation of a system for handling large numbers of novel variants requiring review and classification, and a comprehensive reporting system to meet the needs of patients and their health care providers.

The validation study showed that the 25-gene panel meets the rigorous quality standards necessary to provide useful data in the clinical setting. The results of the 25-gene panel were shown to be equivalent to those obtained using Sanger DNA sequencing analysis. Extensive validation covered the sequencing and large rearrangement components of the assay with suitably large study sets to provide reliable data. 
Table 3 Previously characterized large rearrangements that were included in the study

\begin{tabular}{|c|c|c|c|c|}
\hline Gene & Mutation & microarray CGH & NGS dosage analysis & MLPA \\
\hline \multirow[t]{2}{*}{$A P C$} & deletion promoter $1 \mathrm{~B}$ - exon 3 & detected & detected & $n / a$ \\
\hline & deletion exon 4 & detected & detected & $n / a$ \\
\hline \multirow[t]{8}{*}{ BRCA1 } & duplication promoter $1 \mathrm{~A}$ - exon 2 & detected & detected & $n / a$ \\
\hline & deletion promoter $1 \mathrm{~A}$ - exon 19 & detected & detected & $n / a$ \\
\hline & duplication exons 5 - 7 & detected & detected & $n / a$ \\
\hline & deletion exon 13 & detected & n/a - insufficient volume & $n / a$ \\
\hline & duplication exon 13 & detected & detected & $n / a$ \\
\hline & deletion exons $15-16$ & detected & $n / a-$ insufficient volume & $n / a$ \\
\hline & deletion exons $16-17$ & detected & detected & $n / a$ \\
\hline & deletion exon 24 & detected & detected & $n / a$ \\
\hline$B R C A 2$ & duplication exons 12 - 13 & detected & detected & $n / a$ \\
\hline EPCAM & deletion exons 2 - 3' UTR & detected & detected & $n / a$ \\
\hline \multirow[t]{4}{*}{ MLH1 } & deletion exons 4 - 9 & detected & detected & $n / a$ \\
\hline & deletion exon 13 & detected & detected & $n / a$ \\
\hline & deletion exon 14 & detected & detected & $n / a$ \\
\hline & deletion exon 16 & detected & detected & $n / a$ \\
\hline \multirow[t]{8}{*}{ MSH2 } & deletion exon 1 & detected & detected & $n / a$ \\
\hline & deletion exons 1 - 6 & detected & No Result & $n / a$ \\
\hline & duplication exons 1 - 6 & detected & detected & $n / a$ \\
\hline & deletion exons 1 - 7 & detected & detected & $n / a$ \\
\hline & deletion exons 1 - 8 & detected & detected & $n / a$ \\
\hline & deletion exons 1 - 10 & detected & detected & $n / a$ \\
\hline & deletion exons 8 - 15 & detected & detected & $n / a$ \\
\hline & deletion exon 16 & detected & detected & $n / a$ \\
\hline MSH6 & deletion exons 4 ( $3^{\prime}$ end) - 10 & detected & detected & $n / a$ \\
\hline MUTYH & duplication exon 1 & detected & detected & $n / a$ \\
\hline \multirow[t]{5}{*}{ PMS2 } & deletion exon 10 & $n / a$ & $n / a$ & detected \\
\hline & deletion exons 1 - 5 & $n / a$ & $n / a$ & detected \\
\hline & deletion exons 13 - 14 & $n / a$ & $n / a$ & detected \\
\hline & deletion exons $14-15$ & $n / a$ & $n / a$ & detected \\
\hline & deletion exons 9 - 11 & $n / a$ & $n / a$ & detected \\
\hline
\end{tabular}

The comprehensiveness of this assay was confirmed by leveraging multiple orthogonal methods during the validation. For example, sequencing variations were confirmed with Sanger sequencing (Figure 2) and NGS dosage analysis results were confirmed by microarray CGH (Figure 3). Though improvements in this process may still be made, the NGS system for detection of large rearrangement yielded $100 \%$ concordant results for all the large rearrangements in regions covered by both the RainDance and microarray CGH assays. Furthermore, the ability to assess dosage of amplicons allows users to catch some cases of allelic dropout that could be caused by sequence variants under terminal primers which would not be observable in overlapping amplicons.
There are numerous potential advantages to LR detection via NGS. In addition to lowering the amount of DNA required for multiple assays and, therefore, requiring fewer redraws for patients, it also allows for very specific amplicon placement. This is important when dealing with pseudogenes, which are present in many of the genes in this panel, and means that dosage data from the actual coding bases of interest is retrieved rather than relying on nearby, and often intronic, divergent sequences. Finally, the PCR-based nature of the enrichment could also allow for the detection of Alu insertions that disrupt the PCR amplicons used for enrichment, and cause them to either fail to amplify or to amplify poorly. This is important as Alu insertions have been 
observed in coding regions of many genes and even occur as founder mutations such as the Alu insertion BRCA2 exon 3 (c.156_157insAlu) in Portuguese populations [16]. These mutations are generally not detected by methods such as microarray CGH and MLPA. In addition, large rearrangements in PMS2 and CHEK2 were also assessed using MLPA, and non-pseudogene portions were confirmed against NGS and microarray $\mathrm{CGH}$. This concordance between orthogonal methods confirms the strength of this testing platform.

There are some limitations to the study presented here. First, the validation was limited to blood-derived samples. This restricted, at least initially, the availability of the test to one sample type. In addition, rare variants such as deep intronic sequence changes, and rare large rearrangements such as genomic inversions, may not be identified using the PCR-based target enrichment approach used in this assay.

Use of this gene panel can help define a patient's cancer risk and define management options, including changes in routine surveillance procedures at a cost that is comparable to that of single gene testing $[17,18]$. Importantly, the genes in the panel were selected because they could provide clinically significant data, not just an assessment of risk. The availability of a robust assay for these genetic risk factors that uses a standardized analysis procedure such as the one presented here can facilitate more widespread screening for hereditary cancer syndromes.

\section{Conclusions}

These findings represent a thorough validation of the 25 -gene hereditary pan-cancer panel. The results demonstrate that the NGS panel can be used to screen patients for mutations associated with hereditary cancers in a variety of different tissues with a sensitivity and specificity comparable to that of Sanger sequencing. The NGS panel has the advantage of being able to simultaneously screen for multiple genes from several different patients at the same time. Widespread use of this standardized genetic risk assessment tool could increase the identification of patients at high risk for these cancers and potentially improve care by changing surveillance procedures and/or treatment of malignancies.

\footnotetext{
Abbreviations

CGH: Comparative genomic hybridization; Cl: Confidence interval; LR: Large rearrangements; LRA: Long-range amplicons; MLPA: Multiplex ligation-dependent probe amplification analyses; NGS: Next generation sequencing; PCR: Polymerase chain reaction.

\section{Competing interests}

T Judkins, B LeClair, K Bowles, N Gutin, J Trost J McCulloch, A Murray, J Craft, and B Roa are paid employees of Myriad Genetic Laboratories, Inc. S Bhatnagar, B Wardell, M Bastian, J Mitchell, J Chen, T Tran, D Williams, J Potter, S Jammulapati, M Perry, B Morris, and K Timms are paid employees of Myriad Genetics Inc.
}

\section{Authors' contributions}

TJ contributed to the design of the study, interpretation of the data, and creation of the manuscript; provided final approval for the version to be published; and agreed to be accountable for all aspects of the work. BL contributed to the design of the study and interpretation of the data, provided final approval for the version of the manuscript to be published, and agreed to be accountable for all aspects of the work. KB contributed to the design of the study and interpretation of the data, provided final approval for the version of the manuscript to be published, and agreed to be accountable for all aspects of the work. NG contributed to the design of the study, interpretation of the data, and creation of the manuscript and provided final approval for the version to be published. JT contributed to the design of the study and interpretation of the data and provided final approval for the version to be published. JMc contributed to the design of the study and interpretation of the data and provided final approval for the version to be published. SB contributed to the design of the study, interpretation of the data, and creation of the manuscript and provided final approval for the version to be published. AM contributed to the data collection and creation of the manuscript and provided final approval for the version to be published. JCr contributed to the design of the study, interpretation of the data, and creation of the manuscript and provided final approval for the version to be published. BW contributed to the data collection and creation of the manuscript and provided final approval for the version to be published. MB contributed to the data collection and creation of the manuscript and provided final approval for the version to be published. JMi contributed to the data collection and creation of the manuscript and provided final approval for the version to be published. JCh contributed to the data collection and creation of the manuscript and provided final approval for the version to be published. $\Pi$ contributed to the data collection and creation of the manuscript and provided final approval for the version to be published. DW contributed to the data collection and creation of the manuscript and provided final approval for the version to be published. JP contributed to the data collection and creation of the manuscript and provided final approval for the version to be published. SJ contributed to the data collection and creation of the manuscript and provided final approval for the version to be published. MP contributed to the data collection and creation of the manuscript and provided final approval for the version to be published. BM contributed to the design of the study, interpretation of the data, and creation of the manuscript and provided final approval for the version to be published. BR contributed to the design of the study, interpretation of the data, and creation of the manuscript; provided final approval for the version to be published; and agreed to be accountable for all aspects of the work. KT contributed to the design of the study, interpretation of the data, and creation of the manuscript; provided final approval for the version to be published; and agreed to be accountable for all aspects of the work.

\section{Acknowledgements}

This research was funded by Myriad Genetics, Inc. We acknowledge the efforts of the clinicians and patients who have made this work possible. We also thank past and present Myriad Genetics Laboratories, Inc. laboratory directors, genetic counselors, scientists, statisticians, and laboratory personnel for their ongoing dedication. We would also like to thank Matthew Ryder, Courtney Daniels, Jayson Holladay, Jeremy Schoenberger, Jonathan Stevens, Casey Huff, Aaron Skinner, Troy Suzuki, Neal Africano, Dennis Cook, and Sonia Chen. Medical writing assistance was provided by Robert C. Ristuccia, PhD (Precept Medical Communications) through funding by Myriad Genetics, Inc.

\section{Author details}

${ }^{1}$ Myriad Genetic Laboratories, Inc., Salt Lake City, Utah, USA. ${ }^{2}$ Myriad Genetics, Inc., Salt Lake City, Utah, USA.

Received: 6 August 2014 Accepted: 19 March 2015

Published online: 02 April 2015

\section{References}

1. What are the survival rates for melanoma skin cancer by stage? [http:// www.cancer.org/cancer/skincancer-melanoma/detailedguide/melanomaskin-cancer-survival-rates]. 
2. Pal T, Permuth-Wey J, Betts JA, Krischer JP, Fiorica J, Arango H, et al. BRCA1 and BRCA2 mutations account for a large proportion of ovarian carcinoma cases. Cancer. 2005;104(12):2807-16

3. Zhang S, Royer R, Li S, McLaughlin JR, Rosen B, Risch HA, et al. Frequencies of BRCA1 and BRCA2 mutations among 1,342 unselected patients with invasive ovarian cancer. Gynecol Oncol. 2011;121(2):353-7.

4. Balmana J, Stockwell DH, Steyerberg EW, Stoffel EM, Deffenbaugh AM, Reid JE, et al. Prediction of MLH1 and MSH2 mutations in Lynch syndrome. JAMA. 2006;296(12):1469-78.

5. Klee EW, Hoppman-Chaney NL, Ferber MJ. Expanding DNA diagnostic panel testing: is more better? Expert Rev Mol Diagn. 2011;11(7):703-9.

6. Castera L, Krieger S, Rousselin A, Legros A, Baumann JJ, Bruet O, et al. Next-generation sequencing for the diagnosis of hereditary breast and ovarian cancer using genomic capture targeting multiple candidate genes. Eur J Hum Genet. 2014;22(11):1305-13.

7. Molecular Pathology Checklist: CAP Accreditation Program. [http://www.cap. org/apps/cap.portal?_nfpb=true\&_pageLabel=accreditation]

8. Rehm HL, Bale SJ, Bayrak-Toydemir P, Berg JS, Brown KK, Deignan JL, et al. ACMG clinical laboratory standards for next-generation sequencing. Genet Med. 2013;15(9):733-47.

9. Tung N, Battelli C, Allen B, Kaldate R, Bhatnagar S, Bowles K, et al. Frequency of mutations in individuals with breast cancer referred for BRCA1 and BRCA2 testing using next-generation sequencing with a 25-gene panel. Cancer. 2015;121(1):25-33

10. Kazazian $\mathrm{H}$, Boehm C, Seltzer W. ACMG recommendations for standards for interpretation of sequence variations. Genet Med. 2000;2(5):302-3.

11. Harland M, Holland EA, Ghiorzo P, Mantelli M, Bianchi-Scarra G, Goldstein $\mathrm{AM}$, et al. Mutation screening of the CDKN2A promoter in melanoma families. Genes Chromosomes Cancer. 2000;28(1):45-57.

12. Hitchins MP, Rapkins RW, Kwok CT, Srivastava S, Wong JJ, Khachigian LM, et al. Dominantly inherited constitutional epigenetic silencing of MLH1 in a cancer-affected family is linked to a single nucleotide variant within the 5'UTR. Cancer Cell. 2011;20(2):200-13.

13. Hall MJ, Forman AD, Pilarski R, Wiesner G, Giri VN. Gene panel testing for inherited cancer risk. J Natl Compr Canc Netw. 2014;12(9):1339-46.

14. Hiraki S, Rinella ES, Schnabel F, Oratz R, Ostrer H. Cancer risk assessment using genetic panel testing: considerations for clinical application. J Genet Couns. 2014;23(4):604-17.

15. Kurian AW, Hare EE, Mills MA, Kingham KE, McPherson L, Whittemore AS, et al. Clinical evaluation of a multiple-gene sequencing panel for hereditary cancer risk assessment. J Clin Oncol. 2014;32(19):2001-9.

16. Peixoto A, Santos C, Pinto P, Pinheiro M, Rocha P, Pinto C, et al. The role of targeted BRCA1/BRCA2 mutation analysis in hereditary breast/ovarian cancer families of Portuguese ancestry. Clin Genet. 2014;doi 10.1111/ cge.12441 [Epub ahead of print].

17. Walsh T, Casadei S, Lee MK, Pennil CC, Nord AS, Thornton AM, et al. Mutations in 12 genes for inherited ovarian, fallopian tube, and peritoneal carcinoma identified by massively parallel sequencing. Proc Natl Acad Sci U S A. 2011;108(44):18032-7.

18. Walsh T, Lee MK, Casadei S, Thornton AM, Stray SM, Pennil C, et al. Detection of inherited mutations for breast and ovarian cancer using genomic capture and massively parallel sequencing. Proc Natl Acad Sci U S A. 2010;107(28):12629-33.

\section{Submit your next manuscript to BioMed Central and take full advantage of:}

- Convenient online submission

- Thorough peer review

- No space constraints or color figure charges

- Immediate publication on acceptance

- Inclusion in PubMed, CAS, Scopus and Google Scholar

- Research which is freely available for redistribution 